\title{
AMEROTYPHLOPS MICROSTOMUS (SQUAMATA: TYPHLOPIDAE)
}

Alvaro Monter-Pozos ${ }^{1}$, Pedro E. NAhuat-Cervera ${ }^{2,3 *}$ \& J. Rizieri Avilés-Nove LO ${ }^{4}$

${ }^{1}$ División de Ciencias Biológicas y de la Salud., Universidad Autónoma Metropolitana-Iztapalapa. Av. San Rafael Atlixco 186, Leyes de Reforma 1ra Secc, Del. Iztapalapa, C. P. 09340 . Ciudad de México, México.

${ }^{2}$ Ekuneil Peninsula de Yucatán. Calle 52, número 670. Col. Centro. C.P. 97000. Mérida, Yucatán, México.

${ }^{3}$ Asociación Juvenil de Ciencia Yucatán. Calle 35-C x 60, Fraccionamiento Colonia Buenavista. C.P. 97215. Mérida, Yucatán, México

${ }^{4}$ Campus de Ciencias Biológicas y Agropecuarias, Universidad Autónoma de Yucatán, Km 15.5 carr. Mérida-Xmatkuil, C.P. 97315. Mérida, Yucatán, México.

"Correspondencia:pedro.nahuat4@gmail.com

México: Yucatán: Dzemul, $5.2 \mathrm{~km}$ al sur de la zona arqueológica X'Cambó $\left(21.265197^{\circ} \mathrm{N}, 89.3483^{\circ} \mathrm{O}\right.$, WGS 84; $\left.9 \mathrm{msnm}\right), 18$ de marzo de 2020 a las 09:12 h. Este reporte representa el primer registro de Amerotyphlops microstomus en el municipio de Dzemul, así como el registro más septentrional en la Península de Yucatán, ubicado a $32.4 \mathrm{~km}$ y $32.7 \mathrm{~km}$ de los registros más cercanos, en la localidad de Dzibilchaltún, municipio de Mérida,
Yucatán, México, con número de catálogo FMNH 153590 (GBIF, 2020) y 4481414 (iNaturalist, 2020), respectivamente, y a $7.7 \mathrm{~km}$ de la línea de la costa (Fig. 1). Individuo adulto ( $\mathrm{TL}=345 \mathrm{~mm}$, peso $=8 \mathrm{~g}$, Fig. 2), se encontró muerto con una pequeña lesión en la cabeza, posiblemente generada por algún ave. El cuerpo estaba sobre un camino de terracería rodeado por vegetación secundaria arbórea de selva baja espinosa caducifolia.

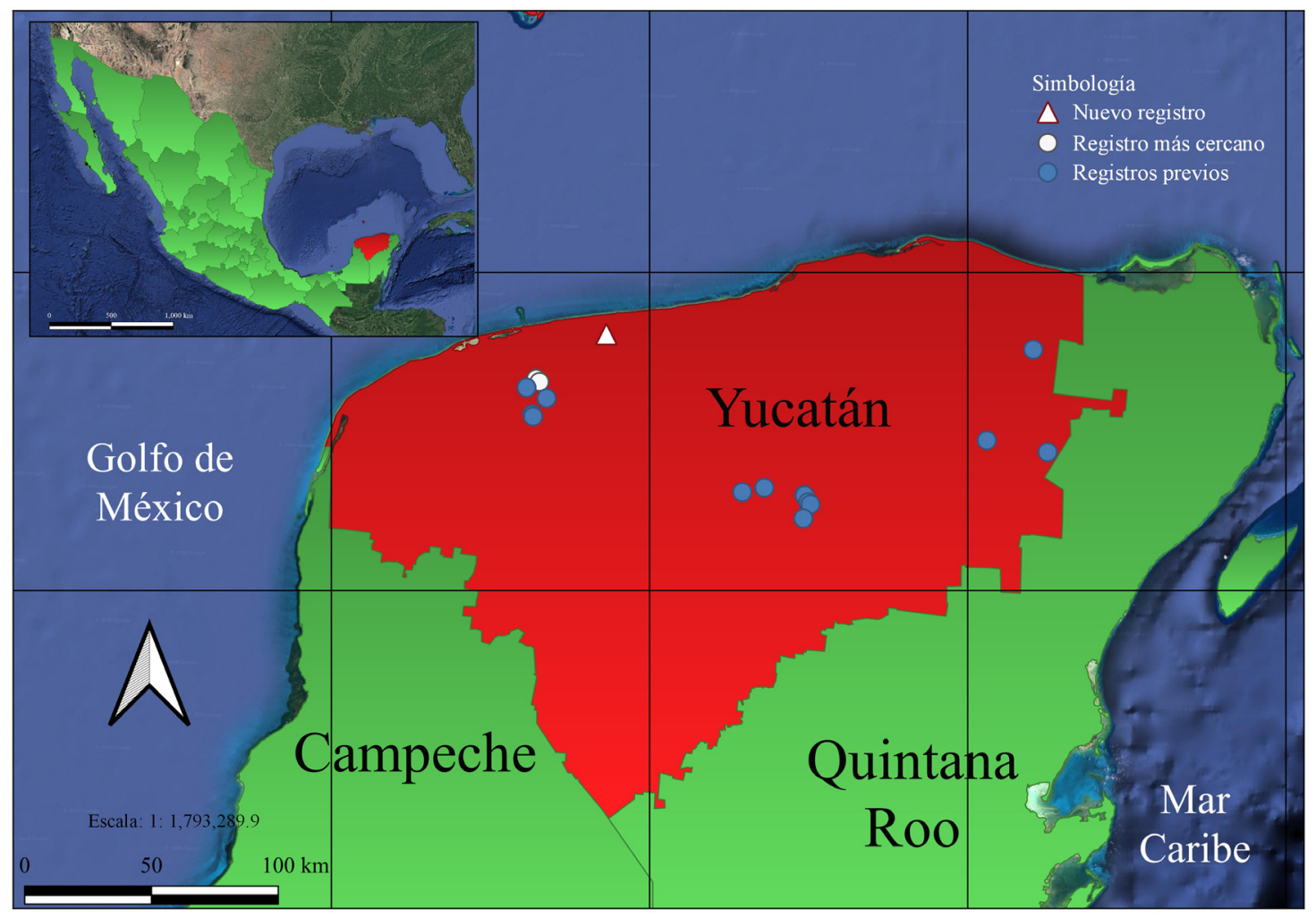

Figure 1. Geographic distribution of Amerotyphlops microstomus in the state of Yucatán, adding the new record of this species in the municipality of Dzemul. Blue circles represent the previous known localities, white circles are the closest known localities and the white triangle is the new record.

Figura 1. Distribución geográfica de Amerotyphlops microstomus en el estado de Yucatán, anexando el nuevo registro en el municipio de Dzemul. Los círculos azules representan las localidades previamente conocidas, los círculos blancos las localidades conocidas más cercanas al nuevo registro y el triángulo blanco el nuevo registro. 


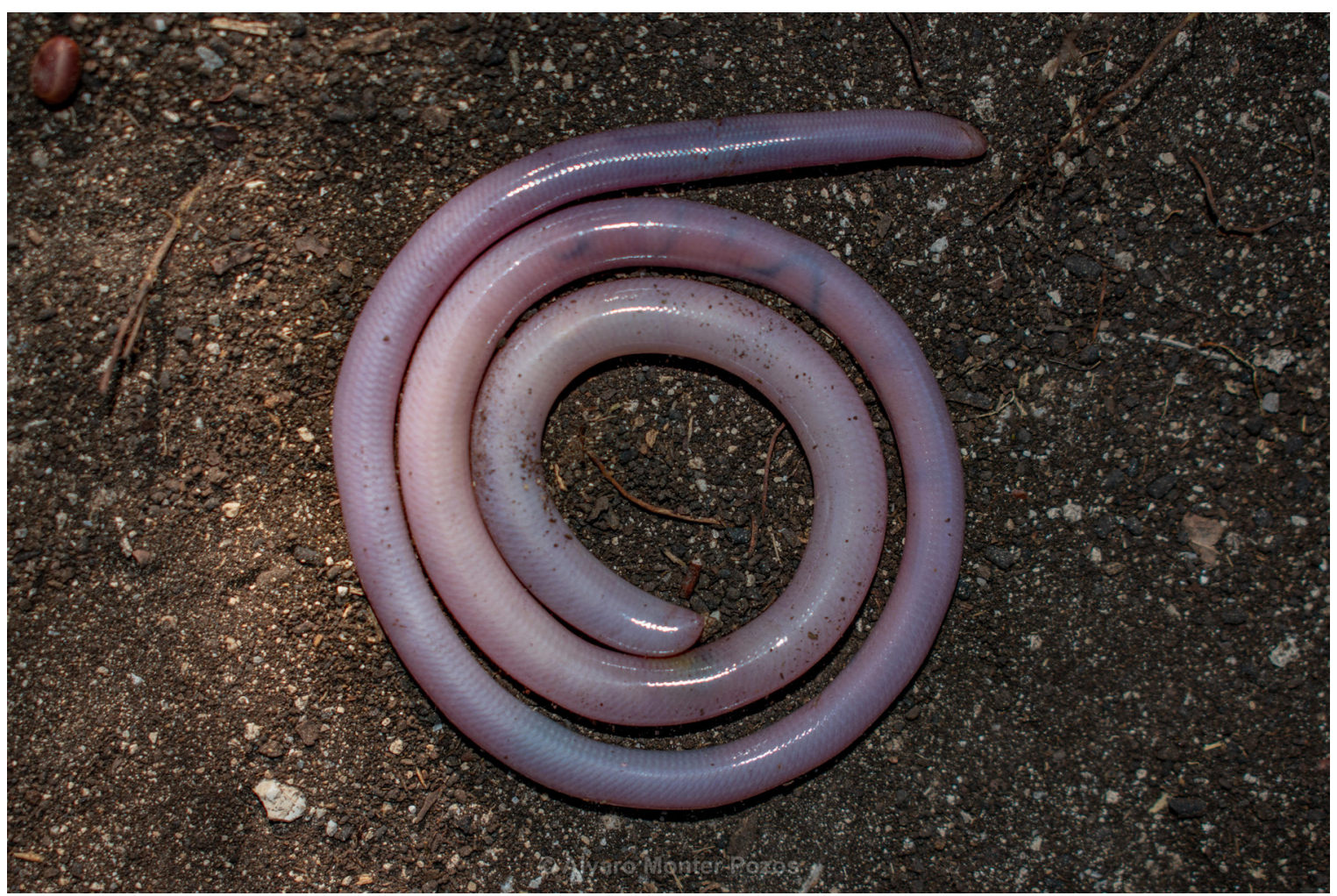

Figure 2. Adut Amerotyphlops microstomus from Dzemul, Yucatán, Mexico. Photograph: Alvaro Monter Pozos.

Figura 2. Ejemplar adulto de Amerotyphlops microstomus encontrado en Dzemul, Yucatán, México. Fotografía: Alvaro Monter Pozos.

Este registro incrementa a seis el número de municipios y a 10 el número de localidades conocidas con registro de $A$. microstomus en el estado de Yucatán (Lee, 1996; GBIF, 2020). El ejemplar fue encontrado y fotografiado por Alvaro Monter Pozos y verificado por José Rogelio Cedeño Vázquez. El registro fotográfico se depositó en la colección digital de The University of Texas at Arlington (UTADC 9563), Texas, USA.

Agradecimientos.- Agradecemos a José Rogelio CedeñoVázquez por la verificación del ejemplare encontrado, y a Gregory G. Pandelis por proporcionar los números de catálogo.

\section{LITERATURA CITADA}

GBIF.org. 2020. Amerotyphlops microstomus occurrence data. https:// doi.org/10.15468/dl.4p7mdk. [Consultado el o2 de abril de 2020]

Lee, J.C. 1996. The amphibians and reptiles of the Yucatán Peninsula. Cornell University Press. USA. 